\title{
Species traits and species diversity affect community stability in a multiple stressor framework
}

\author{
Sabine Flöder ${ }^{1, *}$, Helmut Hillebrand ${ }^{1,2}$ \\ ${ }^{1}$ Aquatic Ecology, Botanical Institute, University of Cologne, Gyrhofstr. 15, 50931 Cologne, Germany \\ ${ }^{2}$ Present address: Plankton Ecology, Institute for Chemistry and Biology of the Marine Environment (ICBM), \\ Carl-von-Ossietzky University Oldenburg, Schleusenstrasse 1, 26382 Wilhelmshaven, Germany
}

\begin{abstract}
Two decades of biodiversity-ecosystem functioning research have not yet led to consensus view on how local dominance and biodiversity affect the stability of ecosystems in the presence of disturbances. As natural systems are usually affected by multiple stressors, we tested the diversity-stability hypothesis on phytoplankton communities subjected to combinations of 2 stressors ( $\mathrm{pH}$ reduction and simulated grazing). Monocultures revealed uncorrelated species' sensitivities to the 2 stressors and the presence of a best-performing species (Scenedesmus obliquus). We thus selected 2 experimental species combinations, either (A) including or (B) excluding $S$. obliquus, and inoculated at 3 diversity levels $(2,4,8$ species). Both stressors induced significant biovolume loss and increased temporal biovolume variation, but the effect on final biomass $\left(B_{\text {tot }}\right)$ differed between combinations and with richness. The fast recovery of $S$. obliquus precluded long-term disturbance effects on $B_{\text {tot }}$ in Combination A. Correspondingly, these communities showed slower recovery rates and lower $B_{\text {tot }}$ with more species present, since the strong performance of $S$. obliquus was diluted by more species being present. More species-rich assemblages in Combination B lost more biovolume after stressor application, whereas the recovery rates showed a more complex pattern, depending on stressor sequence and species richness. Our study suggests that the stability of communities in the face of multiple disturbances cannot be predicted from a general diversity-stability relationship alone, but depends on the correlation between species sensitivities to different stressors and on the population dynamics of a well-performing species when dominant.
\end{abstract}

KEY WORDS: Stability - Species richness - Species identity · Stressor-induced community resistance · Disturbance

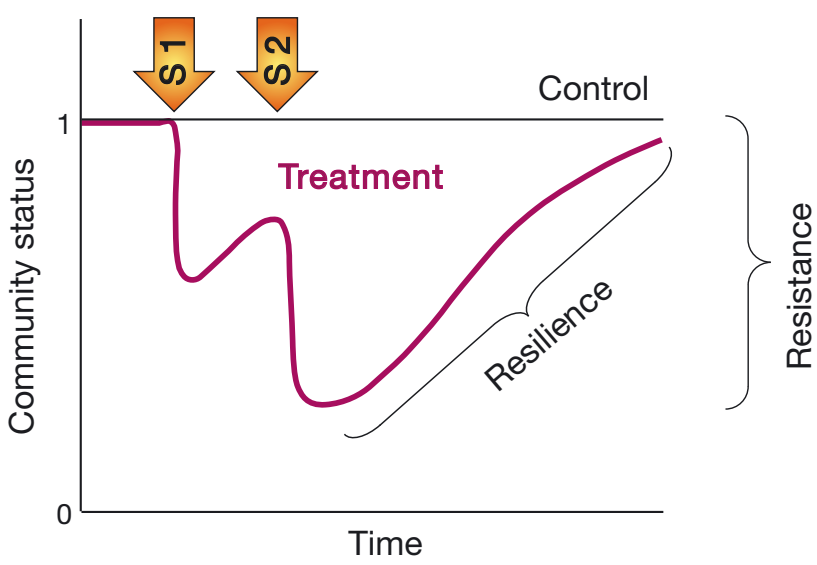

Community stability. Effect of 2 stressors (S1 and S2) on community status. Resilience is determined by the rate of recovery, resistance by the net change in community status

Diagram: H. Hillebrand and S. Flöder

\section{INTRODUCTION}

In the face of unprecedented loss in global diversity, the relationship between diversity and stability has again become a major focus of research in ecology. Whereas evidence accumulates for consistent diversity effects on the magnitude of processes (see e.g. Cardinale et al. 2006, Hillebrand \& Matthiessen 2009), there is ongoing debate as to whether diversity induces certain aspects of stability.

Two aspects of stability have been to date analysed separately, although they are not fully distinct from each other: variability over time and response to disturbance. In the context of the diversity-stability hypothesis (DSH), diversity is suggested to reduce the temporal fluctuation in aggregate ecosystem processes by compensatory dynamics (Tilman 1996, Cot- 
tingham et al. 2001), statistical averaging (Doak et al. 1998), or by decreasing the synchrony of population dynamics (Roscher et al. 2011). This aspect of the DSH is comparably well underpinned by theory, e.g. by the insurance model (Yachi \& Loreau 1999), which bases diversity effects on the fact that diverse communities are more likely to show compensatory dynamics than communities of low biodiversity. Although compensatory dynamics in response to environmental fluctuation has been analysed in natural systems (Vasseur \& Gaedke 2007, Winfree \& Kremen 2009) and experiments (Gonzalez \& DescampsJulien 2004, Downing 2008), there is considerable debate as to whether compensatory dynamics generally prevail in ecosystems (Houlahan et al. 2007, Valone \& Barber 2008).

Regarding the second aspect, the response to disturbance, it has been suggested that the importance of biodiversity for community response to a pulse perturbation depends on the resistance (the ability to withstand disturbance) and resilience (the ability to recover from disturbance) of the species within the community (Pfisterer \& Schmid 2002, van Ruijven \& Berendse 2010). In this context, disturbance is defined as a temporally explicit phenomenon that reduces community biomass by causing its partial or total destruction (sensu Grime 1979). The prediction of how biodiversity affects these 2 aspects of stability is not straightforward as each potentially requires different species traits. These traits enable the species to resist or tolerate the disturbance or to grow rapidly after the disturbance. Trade-offs between growth and defendedness can be expected (Fine et al. 2006), as adaptations to resist disturbances potentially come at a cost to growth rate, which defines whether a species can contribute quickly to recovery. The relative proportion of sensitive, tolerant and resistant species may, therefore, foster different diversity-stability relationships in the response to disturbance. Consequently, experimental studies (e.g. Pfisterer \& Schmid 2002, Moorthi et al. 2008) remained inconclusive with respect to the presence of general biodiversity effects on stability following pulse perturbations.

The overall relationship between biodiversity and stability after disturbance will thus depend on how the traits that relate to resistance and recovery are distributed within the community. This suggests that the dominance structure (evenness) of communities may be as important for community stability as richness (Hillebrand et al. 2008, Sasaki \& Lauenroth 2011). We propose that population dynamics of dominant species (low evenness) determine the response of the entire community, if these species display high resistance or resilience. If dominant species are highly sensitive and recover slowly, compensatory growth of rare species is needed to maintain or recover high levels of ecosystem performance. In this case, higher evenness increases stability. Thus, the relationship between diversity and response to disturbance is largely determined by interdependencies between the number of species present and their identity and degree of dominance.

In addition to the unclear separation between different aspects of stability and diversity, studies within the framework of the DSH have focussed entirely on the effects of single disturbances, although natural systems are commonly affected by multiple aspects of disturbance (Liiri et al. 2002). As stressors (= disturbances) are expected to potentially interact with non-additive consequences for biomass reduction, the stability of communities that are subjected to multiple stressors cannot be extrapolated from studies investigating single stressors (Breitburg et al. 1998). Vinebrooke et al. (2004) proposed 3 scenarios of multiple stressor effects in the framework of the DSH: species' tolerances to multiple stressors may be randomly distributed, or they may be positively or negatively correlated. A positive correlation would result in higher stability, because species that survive the impact of Stressor A are likely to tolerate Stressor B (stressor-induced community tolerance). Conversely, a negative correlation between species' tolerances to multiple stressors would lead to a strong decrease in stability (stressor-induced community sensitivity). If species' tolerances to stressors are randomly distributed and unrelated, exposure to Stressor A is not likely to influence the effect of Stressor B.

The objective of our study was to investigate the effects of species richness and species identity on the stability of communities that are affected by multiple stressors. We performed 2 experiments using various strains of freshwater phytoplankton affected by combinations of 2 artificial stressors: $\mathrm{pH}$ reduction and grazing. First, we analysed the response of these strains to both stressors, as well as their growth rates, in a monoculture experiment. In the subsequent diversity experiment, we simulated 2 different scenarios of community composition using a combinatorial design. The first scenario (Combination A) focussed on the hypothesis that stability depends solely on the population dynamics of a dominant species with functional traits allowing its persistence after disturbance. This species was present at all levels of species richness $(2,4$ and 8 species). The 
second scenario (Combination B) tested the hypothesis that, in the absence of such a dominant and highly functional species, the richness of species determines the response of a community to multiple stressors.

\section{MATERIALS AND METHODS}

\section{Experimental conditions}

Eleven phytoplankton strains represented the species pool of our study: Cylindrospermum sp. (Cyl), Cyclotella sp. $\left(C_{y c}\right)$, Melosira sp. (Mel), Fragilaria capucina (Fra), Cryptomonas pyrenoidifera (Cry), Tetraselmis sp. (Tes), Chlamydomonas tericola (Chl), Pediastrum sp. (Ped), Oocystis sp. (Ooc), Tetraedron minimum (Tee), Scenedesmus obliquus (Sce), see Supplement 1 at www.int-res.com/articles/suppl/ b017p197_supp.pdf for further information). Algae were grown using a modified WC medium (Guillard 1975). The concentrations of the most important nutrients for phytoplankton growth $\left(50 \mu \mathrm{g} \mathrm{P}^{-1}, 1000 \mu \mathrm{g}\right.$ $\mathrm{N}^{-1}$ and $1500 \mu \mathrm{g} \mathrm{Si}^{-1}$ ) corresponded to a eutrophic lake with phosphorus limitation. The medium was buffered to maintain a $\mathrm{pH}$ of 7 . Experimental communities were grown in semi-continuous culture using Erlenmeyer flasks (total culture volume [ $\left.V_{\text {tot }}\right]$ : $152 \mathrm{ml}$ ). Every other day, $38 \mathrm{ml}$ of $V_{\text {tot }}$ was replaced with new medium $\left(V_{\text {new }}\right)$, resulting in a replacement rate $(D)$ of $0.125 \mathrm{~d}^{-1}\left(D=V_{\text {new }} / V_{\text {tot }}\right)$. Day:night cycle $(16: 8 \mathrm{~h})$, light intensity $\left(140 \pm 5 \mu \mathrm{mol} \mathrm{m} \mathrm{m}^{-2} \mathrm{~s}^{-1}\right.$ photosynthetic photon flux density) and experimental temperature $\left(20^{\circ} \mathrm{C}\right)$ were kept constant. A shakertable $(<100 \mathrm{rpm})$ kept the cultures in suspension. All culture work was done under sterile conditions.

The 2 stressors employed in our study were not meant to mimic naturally occurring disturbances but to impose 2 sources of mortality, potentially requiring different adaptations and thus allowing for trade-offs in species tolerances to either of the 2 stressors (see 'Introduction'). pH reduction was a chemical stressor, whereas simulated grazing was a mechanical disturbance imposing strong size-selective mortality. For pH-reduction we used sterilised $\mathrm{HCl}$ and $\mathrm{NaOH}$ solutions $(1 \mathrm{~N})$. The $\mathrm{pH}$ was measured, reduced to $\mathrm{pH} 5$, and on the following day it was set back to its original value. Since it was not possible to determine $\mathrm{pH}$ under sterile conditions, we prepared extra cultures for each experimental treatment that experienced $\mathrm{pH}$ reduction. These were cultured under the same conditions as the replicates of the treatments. To simulate grazing, we filtered $30 \%$ of the algal suspension through a $5 \mu \mathrm{m}$ mesh-size membrane under sterile conditions. We chose this mesh size because it could be expected to affect small and single-celled strains to a lesser extent than larger and filamentous strains.

\section{Experimental design and analyses}

To test the effect of multiple stressors on phytoplankton communities of different diversity, we conducted 2 experiments. The monoculture experiment was designed to test how each single species in our species pool responded to the stressors $\mathrm{pH}$ reduction $(\mathrm{pH})$ and grazing (gra). In the diversity experiment we exposed communities of different diversity and species composition to 2 stressors in different combinations (gra-gra, gra- $\mathrm{pH}, \mathrm{pH}-\mathrm{gra}, \mathrm{pH}-\mathrm{pH}$ ).

In the monoculture experiment, each of the strains in our species pool was grown as single species culture. To gain an inoculum, the strains were grown as batch cultures under the experimental conditions described above for $5 \mathrm{~d}$ prior to the start of the experiment. For each strain, the same biovolume $(1.82 \times$

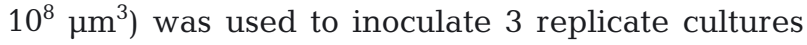
per treatment (gra, $\mathrm{pH}$, control; $11 \times 3 \times 3=99$ experimental units). Duration of the monoculture experiment was $14 \mathrm{~d}$. To account for an initial growth phase in the monoculture experiment, single stressors (either gra or $\mathrm{pH}$ ) were applied on Day 7. Samples for microscopic analysis were taken before stressor application (Day 7), and on Days 8, 11 and 14. Water samples for phosphorus analysis were taken at the end of the experiment.

The diversity experiment consisted of multi-species cultures of different species richness in 2 scenarios. These were separated by the presence or absence of a 'super-species' with certain trait combinations for which we had clear expectations of its effect on the response to disturbance. Within the 2 scenarios, richness levels of the experimental communities were 2 , 4 and 8 species. The lower richness levels were always nested subsets of the 8-species assemblage, as this was the only way to simulate the loss of richness while still keeping a certain best-performing species. It has been argued that nested subsetting restricts the test of species richness effects to certain species assemblages and thus does not represent a test of a general diversity-function relationship (Huston 1997). However, the alternative approach to random subsetting has also been questioned as it makes more speciose assemblages too similar in species composition compared to those which are less speciose, and this can strongly affect the variability of biomass production at different richness levels (Fukami 2001). 
In Combination $\mathrm{A}, \mathrm{Sce}$, the species that (based on initial growth rate, competitive ability, biovolume losses and recovery rates) had been identified as the best-performing species in our monoculture experiment (see 'Results: Monoculture experiment' and Supplement 2 at www.int-res.com/articles/suppl/ b017p197_supp.pdf), was present at each richness level, whereas the other species were randomly chosen from our species pool. The species in Combination B were a random selection from the pool after Sce had been excluded (2 species: Sce and Ooc [A] or Cry and Mel [B]; 4 species: Sce, Ooc, Tee and Chl [A] or Cry, Mel, Tes and Cyc [B], 8 species: Sce, Ooc, Tee, Chl, Cry, Mel, Tes and Ped [A] or Cyl, Cyc, Tee, Fra, Tes, Cry, Mel and Ped [B]). To obtain an inoculum, we grew each species in batch culture as described for the monoculture experiment. Multi-species cultures

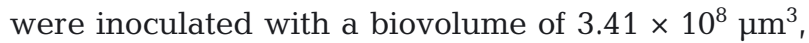
where the total inoculate biovolume consisted of equal fractions that corresponded to the number of species ( 2 species: $2 \times\left[1.705 \times 10^{8} \mu \mathrm{m}^{3}\right], 4$ species: $4 \times$

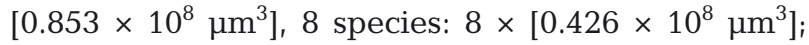
evenness $=1$ ). Experimental communities were exposed to different stressor combinations (gra-gra, gra-pH, $\mathrm{pH}-\mathrm{gra}, \mathrm{pH}-\mathrm{pH}$ ) or remained undisturbed (control). Each treatment was replicated twice, which resulted in $60(2 \times 3 \times 5 \times 2)$ experimental units. The average duplication time of the species in our pool was $2 \mathrm{~d}$ during the initial growth phase (Days 0 to 7 ) of the monoculture experiment. To allow for 15 microalgal generations, we chose $31 \mathrm{~d}$ for the duration of the experiment. This duration is shorter than the time span (35 to $50 \mathrm{~d}$ ) in which the occurrence of competitive exclusion can be expected in phytoplankton communities cultured under semi-continuous conditions (Flöder \& Burns 2005). It is likely, therefore, that the communities in our experiments were in a transitional stage (see Supplement 5 at www.int-res.com/articles/ suppl/b017p197_supp.pdf for population dynamics). Experimental communities in the diversity experiment were exposed to the first disturbance on Day 7 and to the second disturbance on Day 13. To monitor the effect of the stressors on the communities, the experiment was sampled immediately before and after stressor application on Days 7, 8, 13 and 14. Further sampling was performed on Days 17, 27 and 31.

\section{Sample analyses}

Analysis of dissolved soluble reactive phosphate (SRP) followed the method by Strickland \& Parsons (1978). In the monoculture experiment, the final con- centration of SRP in control treatments was used as an indicator for competitive ability (comparable to Tilman's [1982] limiting resource threshold $R^{*}$ in cultures at carrying capacity).

Algal abundance was analysed microscopically counting at least 400 cells. The dimensions of 20 individuals of each species were determined to calculate the average specific biovolume (Hillebrand et al. 1999). These data were used to calculate final total biovolume $\left(B_{\text {tot }}\right)$, its temporal coefficient of variation $\left(\mathrm{CV}_{\mathrm{B}}\right)$ and the evenness index $\left(E_{\mathrm{B}}\right)$ at the end of the experiment:

$$
\begin{gathered}
E_{\mathrm{B}}=\frac{H_{\mathrm{B}}{ }^{\prime}}{H_{\mathrm{B} \max }^{\prime}} \\
H_{\mathrm{B}}{ }^{\prime}=-\sum p_{i} \ln \left(p_{i}\right)
\end{gathered}
$$

where $H_{\mathrm{B}}{ }^{\prime}$ denotes the Shannon index, $H_{\mathrm{B}}{ }^{\prime}$ max denotes the theoretical diversity maximum $(=\ln$ (species richness)) and $p_{i}$ is the relative proportional contribution of species $i$ to total biovolume (Washington 1984).

Growth rates were calculated based on biovolume according to:

$$
\mu=\frac{\ln \left(B_{2}\right)-\ln \left(B_{1}\right)}{t_{2}-t_{1}}
$$

where $\mu$ denotes the specific growth rate per day, $t_{1}$ and $t_{2}$ are 2 points in time during the course of the experiment and $B_{1}$ and $B_{2}$ denote the total community biovolume (diversity experiment) or the biovolume of 1 species (monoculture experiment) at $t_{1}$ and $t_{2}$, respectively.

To specifically analyse the resistance and resilience of the experimental communities, percent biovolume loss and recovery rate were calculated. In the monoculture experiment, biovolume in the disturbed treatments was expressed as a percentage of the average biovolume in the control for the respective strain. In the diversity experiment, biovolume in the disturbed treatments was expressed as a percentage of the biovolume in the control for the respective species composition, by which we randomly aligned the 2 replicates of treatment and control. Population and community losses due to disturbance were calculated for the day following the final disturbance (Day 14), and high resistance is reflected by low percentages of biovolume loss. The relative biovolume (i.e. in relation to the undisturbed control) was also employed to estimate recovery rates from specific growth rates after the final disturbance (Day 14). We calculated the rate of biovolume increase between Day 14 and the recovery date, which we defined as the day when the post-disturbance biovolume 
reached the biovolume of undisturbed control cultures on Day 14. Exact recovery dates had to be calculated, since post-disturbance growth rates tended to change over time (see Supplement 3 at www.intres.com/articles/suppl/b017p197_supp.pdf for details).

\section{Statistical analyses}

In the monoculture experiment, we tested the hypothesis that species differ in growth rate and percentage loss by a 1 -factor ANOVA followed by a post hoc test (Tukey's HSD). Species identity was the sole independent factor, though dependent variables consisted of growth rate and final remaining SRP, as well as the percentage of biovolume loss, the recovery rate and the recovery time for $\mathrm{pH}$ reduction and simulated grazing. Correlation analyses were used to test whether the recovery rates and the specific loss of biomass due to $\mathrm{pH}$ reduction and grazing were related.

In the diversity experiment, we performed separate factorial ANOVAs for Combinations A (Sce present) and B (Sce absent), as these combinations tested different hypotheses which would have been masked by a nested design (combination would have to be nested in richness) of a combined analysis. In Combination A, we tested whether the presence of Sce was sufficient to maintain resistance and resilience independent of the richness of the surrounding community. In Combination B, we tested whether more species were needed to maintain stability in the absence of a strongly performing species. The factorial ANOVA comprised richness and stressor regime as factors, and final biovolume, temporal biovolume variation, $E_{\mathrm{B}}$, percent biovolume loss, recovery rate and co-tolerance index as response variables. The analysis of species' co-tolerance to multiple stressors was based on the number of growing species recorded after the disturbances (see Supplements 4 \& 5 at www.int-res.com/articles/suppl/b017p197_supp.pdf for details). For a diversity-independent measure, we subtracted the number of species that displayed positive growth rates after the application of Stressor 1 from the number of species that continued to grow after the application of Stressor 2. This difference was divided by the number of species recorded prior to the disturbances. Since the difference in the number of growing species can be either positive or negative, the resulting index of stressor-induced community co-tolerance (co-tolerance index) stretches from -1 to +1 , where scores $>0$ indicate stressor-induced community tolerance. Scores between 0 and -0.5 indicate randomly distributed species co-tolerances, and scores below -0.5 point towards stressor-induced community sensitivity.

\section{RESULTS}

\section{Monoculture experiment}

In the monoculture experiment, we observed significant differences between species traits concerning competitive ability, as well as resistance and resilience in response to the stressors $\mathrm{pH}$ reduction and grazing. Initial growth rates ranged from $0.086 \mathrm{~d}^{-1}$ (Tes) to $0.602 \mathrm{~d}^{-1}$ (Sce). Sce, the fastest growing species, also displayed a high competitive ability by reducing the SRP concentration to a low

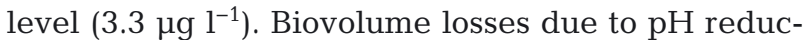
tion ranged from $1.52 \%$ (Ped) to $45.8 \%$ (Cry), and losses due to grazing ranged from $18.1 \%$ (Fra) to $49.5 \%$ (Tes). The species-specific losses in biovolume from $\mathrm{pH}$ reduction and grazing were uncorrelated $(\mathrm{r}=0.14, \mathrm{p}=0.68, \mathrm{~N}=11)$, whereas the recovery rates were positively correlated $(r=0.83, p=0.002)$. Based on its high initial growth rate and competitive ability, the low biovolume loss due to $\mathrm{pH}$ reduction $(17.0 \%)$ and grazing $(23.5 \%)$ and the capability to recover within $5 \mathrm{~d}$ (recovery rate $-\mathrm{pH}$ : 0.038, gra: 0.053), we identified $S c e$ as the best-performing species in our species pool (see Supplement 2 for detailed results).

\section{Community biovolume and evenness in the diversity experiment}

In the diversity experiment, the factors species richness and stressor regime affected $B_{\text {tot }}$ in both species combinations, but at least partly with opposing signs. In Combination $A$, final $B_{\text {tot }}$ decreased with species richness (Table 1, Fig. 1), whereas the stressor regime had no significant effect on $B_{\text {tot }}$. Thus, the presence of $S c e$ resulted in non-significant stressor effects, but this effect of a high-performing species was diluted at higher species richness. Correspondingly, we found significant stressor $\times$ richness interaction, because the negative effect of richness on $B_{\text {tot }}$ was found in disturbed treatments only. In Combination $B$, increasing species richness increased $B_{\text {tot }}$ in the control. $B_{\text {tot }}$ was significantly reduced in comparison to the control when grazing (gra-gra and $\mathrm{pH}$-gra) was the second stressor (Tukey's HSD). When $\mathrm{pH}$ reduction was applied as 
the second stressor ( $\mathrm{pH}-\mathrm{pH}$ and gra- $\mathrm{pH}$ ) treatment, $B_{\text {tot }}$ did not differ from the control. Significant stressor $\times$ richness interaction showed that the increase in $B_{\text {tot }}$ was restricted to the control and those stressor regimes reducing $B_{\text {tot }}$ (gra-gra and $\mathrm{pH}$-gra), whereas richness reduced $B_{\text {tot }}$ in the stressor combination $\mathrm{pH}-\mathrm{pH}$.

Independent of the multiple stressor scenarios applied, disturbance always significantly increased the temporal variability of biovolume in both species combinations (Fig. 1, Table 1). Control treatments showed the lowest $\mathrm{CV}_{\mathrm{B}}$ throughout. In Combination $\mathrm{A}, \mathrm{CV}_{\mathrm{B}}$ increased with species richness (significant main effect; Table 1); thus, more species-rich assemblages showed higher variation of biovolume through time. In Combination B, we found a significant interaction between richness and stressor regime which reflected a strong positive richness
Table 1. Factorial ANOVA results for species combinations A and B: effects of stressor regime (stress) and species richness on biovolume $\left(B_{\text {tot }}\right)$, coefficient of variation $\left(\mathrm{CV}_{\mathrm{B}}\right)$ and biovolume evenness $\left(E_{\mathrm{B}}\right)$ at the end of the experiment. Degrees of freedom in the error term were 15. $B_{\text {tot }}$ data of Combination B failed to become homoscedastic. Since data transformation increased the level of heteroscedasticity, we used untransformed data for the analysis

\begin{tabular}{|lcrrrrrrr|}
\hline Effect & df & \multicolumn{2}{c}{$B_{\text {tot }}$} & \multicolumn{2}{c}{$C^{2} V_{\mathrm{B}}$} & \multicolumn{2}{c|}{$E_{\mathrm{B}}$} \\
& & $F$ & $\mathrm{p}$ & $F$ & $\mathrm{p}$ & $F$ & $\mathrm{p}$ \\
\hline Combination A & & & & & & & \\
Richness & 2 & 15.16 & 0.0003 & 8.688 & 0.0031 & $109.9<0.0001$ \\
Stress & 4 & 0.510 & 0.7293 & $15.50<0.0001$ & 8.133 & 0.0011 \\
Stress $\times$ Richness & 8 & 3.122 & 0.0274 & 2.387 & 0.0697 & 4.038 & 0.0097 \\
Combination B & & & & & & & \\
Richness & 2 & 5.525 & 0.0159 & 1.179 & 0.3347 & $58.20<0.0001$ \\
Stress & 4 & $32.28<0.0001$ & 11.82 & 0.0002 & $51.26<0.0001$ \\
Stress $\times$ Richness & 8 & 3.556 & 0.0165 & 6.181 & 0.0013 & $34.59<0.0001$ \\
\hline
\end{tabular}

effect on $\mathrm{CV}_{\mathrm{B}}$ only for the gra-pH combination. For none of the stressor regimes did we observe a reduction in variability with richness.
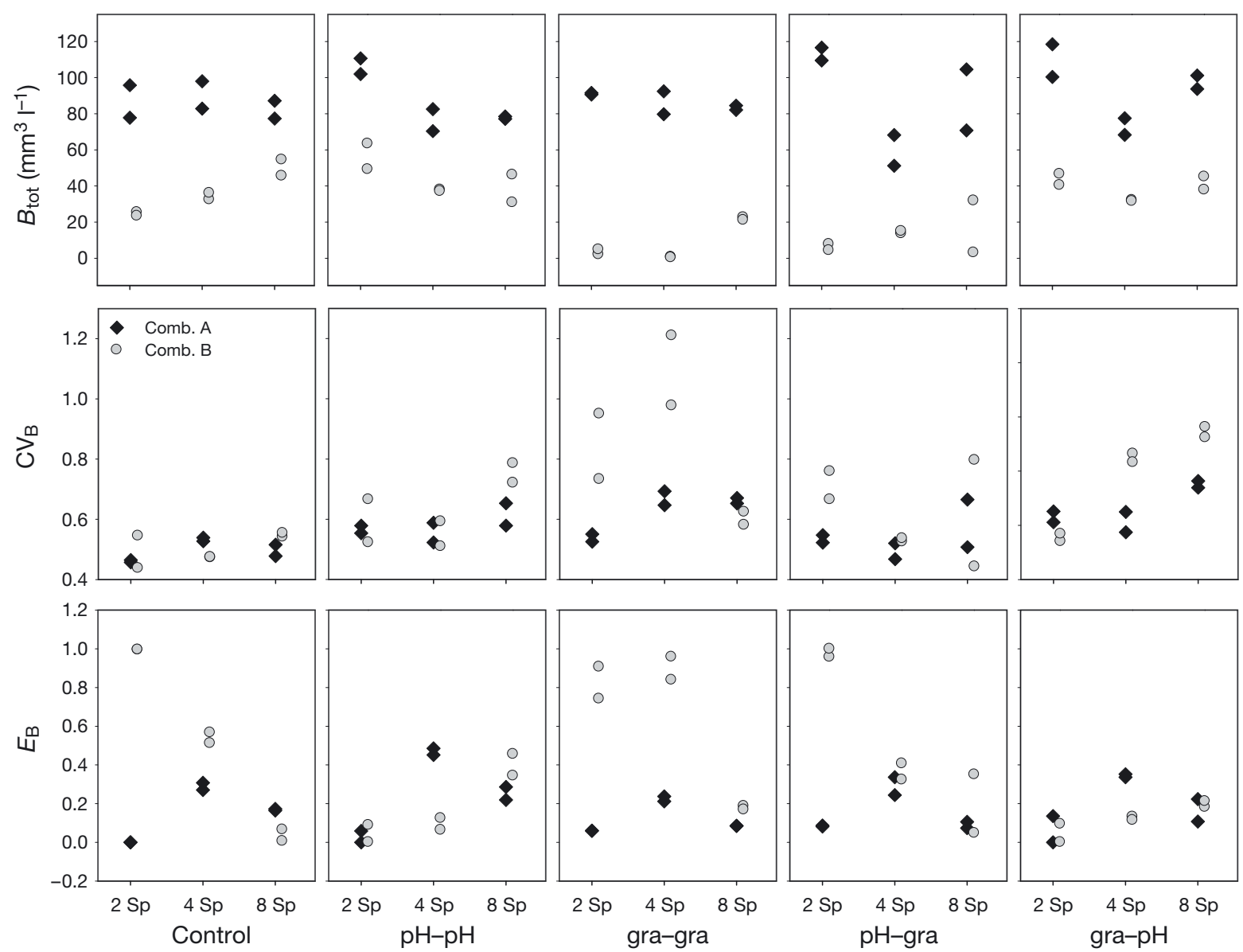

Fig. 1. Total biovolume $\left(B_{\text {tot }}\right)$ at the end of the diversity experiment, temporal coefficient of biovolume variation $\left(\mathrm{CV}_{\mathrm{B}}\right)$ and final biovolume-based evenness $\left(E_{\mathrm{B}}\right)$ in species combinations $\mathrm{A}(\bullet)$ and $\mathrm{B}(\mathrm{O})$ in different combinations of 2 stressors (grazing [gra] and $\mathrm{pH}$ ); 2, 4, and 8 species (Sp) denote levels of species richness being tested 

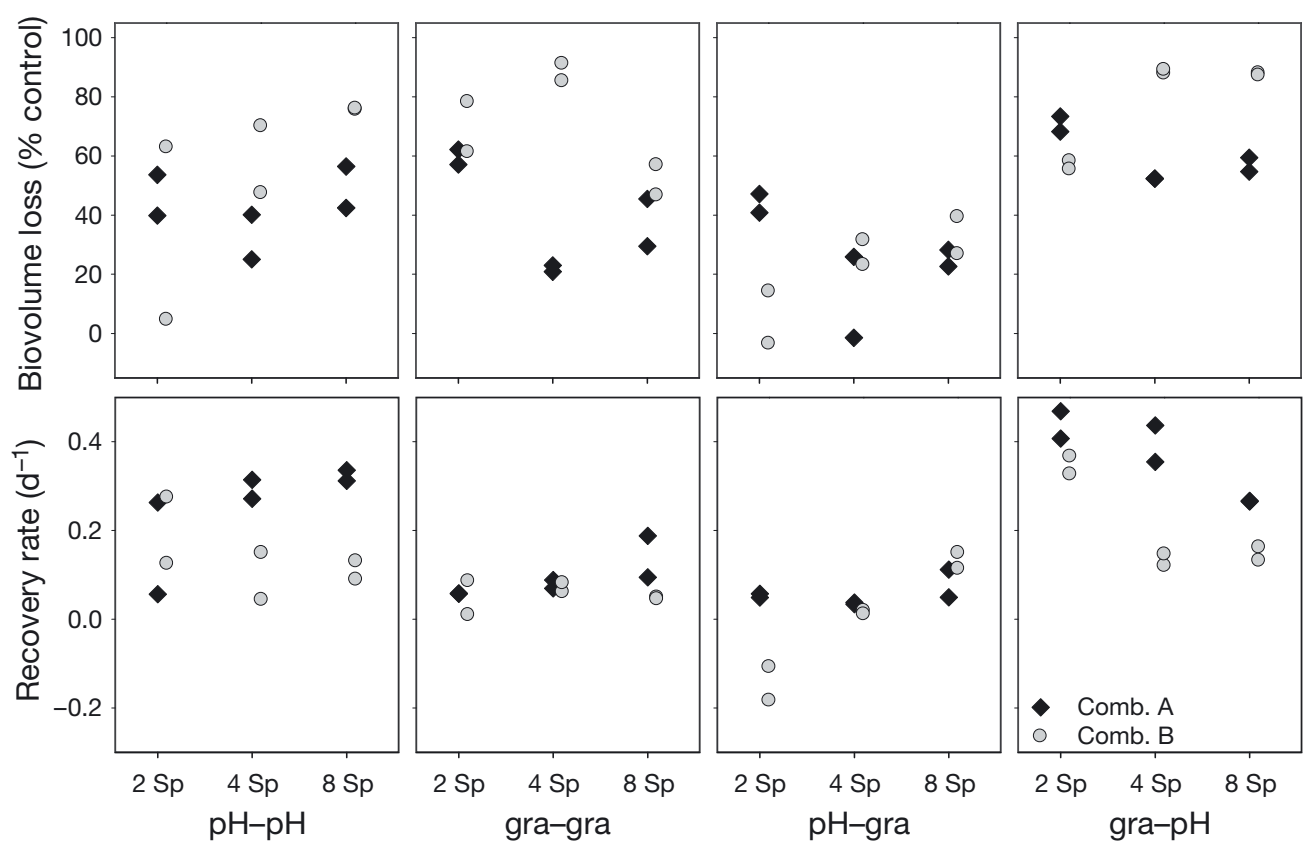

Fig. 2. Total biovolume loss due to multiple stressors and community recovery rate after the second disturbance in species combinations $\mathrm{A}(\bullet)$ and $\mathrm{B}(\mathrm{O})$ in different combinations of 2 stressors (grazing [gra] and $\mathrm{pH}$ ); 2, 4, and 8 species (Sp) denote levels of species richness being tested

Species richness explained $90 \%$ of the variance in $E_{\mathrm{B}}$ in Combination A (Fig. 1, Table 1). $E_{\mathrm{B}}$ at the end of the experiment was lowest for 2 species, highest for 4 species and intermediate in treatments containing 8 species. The stressor regime affected $E_{\mathrm{B}}$ levels, but not its unimodal response to richness. In Combination $\mathrm{B}, E_{\mathrm{B}}$ decreased with increasing richness (significant main effect; Table 1), but with strong interaction with the stressor regime. $E_{\mathrm{B}}$ increased with richness under the stressor regimes $\mathrm{pH}-\mathrm{pH}$ and gra- $\mathrm{pH}$, but decreased in the control and under gra-gra and $\mathrm{pH}$-gra disturbance regimes (significant 2-way interaction; Fig. 1, Table 1).

\section{Community resistance, resilience and co-tolerance in the diversity experiment}

Species richness and stressor regime affected percent biovolume loss in both species combinations (Fig. 2). In both $\mathrm{A}$ and $\mathrm{B}$, high biovolume loss was recorded for the stressor regime gra- $\mathrm{pH}$, whereas the lowest percentage of biovolume was lost under the opposite regime, $\mathrm{pH}$-gra. This result indicates that not only the type of disturbance but also its sequence is highly important for its effect. In Combination A, the percentage of biovolume loss decreased with richness (significant main effect; Table 2), with 4 and 8 species levels losing less biovolume due to disturbance than 2 species levels. The opposite result was found for Combination B, where 4 and 8 species levels lost more biovolume than 2 species levels (significant main effect; Table 2). Diverging from the overall trend, percent biovolume loss in Combination B did not increase with richness under the stressor regime gra-gra (significant 2-way interaction; Table 2).

Table 2. Factorial ANOVA results for species combinations A and B: effects of stressor regime (stress) and species richness on percent biovolume loss, recovery rate and co-tolerance index. Degrees of freedom in the error term were 12. Squared data $\left[X^{\prime}=(X+4)^{2}\right]$ were used for percent biovolume loss. Co-tolerance data were used untransformed because they were homoscedastic. Recovery rates of Combination A failed to become homoscedastic. Since data transformation increased the level of heteroscedasticity, we used untransformed data for the analysis

\begin{tabular}{|c|c|c|c|c|c|c|c|}
\hline \multirow[t]{2}{*}{ Effect } & \multirow[t]{2}{*}{ df } & \multicolumn{2}{|c|}{ Biovolume loss (\%) } & \multicolumn{2}{|c|}{ Recovery rate } & \multicolumn{2}{|c|}{ Co-tolerance } \\
\hline & & $F$ & $\mathrm{p}$ & $F$ & $\mathrm{p}$ & $F$ & $\mathrm{p}$ \\
\hline \multicolumn{8}{|l|}{ Combination A } \\
\hline Richness & 2 & 24.46 & 0.0001 & 0.573 & 0.5786 & 4.694 & 0.0312 \\
\hline Stress & 3 & 22.39 & $<0.0001$ & 44.14 & $<0.0001$ & 0.639 & 0.6042 \\
\hline Stress $\times$ Richness & 6 & 1.918 & 0.1587 & 4.004 & 0.0196 & 3.157 & 0.0427 \\
\hline \multicolumn{8}{|l|}{ Combination B } \\
\hline Richness & 2 & 9.396 & 0.0035 & 1.211 & 0.3318 & 5.747 & 0.0178 \\
\hline Stress & 3 & 26.19 & $<0.0001$ & 22.60 & $<0.0001$ & 2.533 & 0.1062 \\
\hline Stress $\times$ Richness & 6 & 5.170 & 0.0077 & 11.13 & 0.0003 & 0.723 & 0.6397 \\
\hline
\end{tabular}




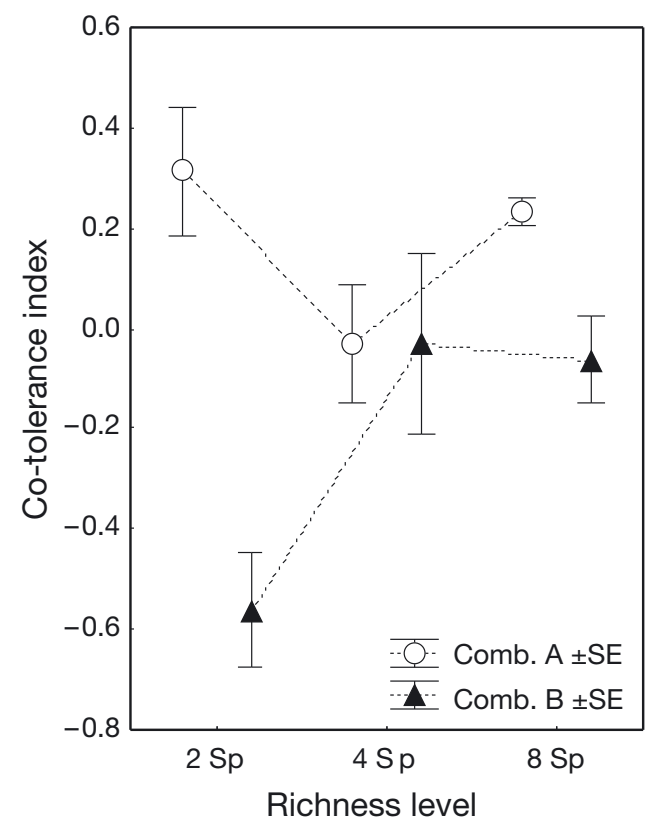

Fig. 3. Co-tolerance index in species combinations A and B. Dotted lines serve to provide orientation only

The stressor regime affected recovery rates in both species combinations (Fig. 2, Table 2). Stressor regimes leading to strong biovolume reduction tended to show the highest recovery rates (gra-pH in $\mathrm{A}$ and $\mathrm{B}_{i} \mathrm{pH}-\mathrm{pH}$ in $\mathrm{A}_{i}$ Fig. 2). Richness had no significant main effect, but we found significant richness $\times$ stressor interactions in both combinations (Table 2). In Combination $\mathrm{A}$, the recovery rate decreased with richness in the gra-pH regime, whereas, under the other disturbance regimes, it tended to increase with richness or remained unchanged (Fig. 2). Recovery rates in Combination $\mathrm{B}$ decreased with richness under stressor regimes gra-pH and $\mathrm{pH}-\mathrm{pH}$, but increased with richness under the $\mathrm{pH}$-gra regime.

The co-tolerance index indicated stressor-induced community tolerance in all treatments of Combination A ( 2 and 8 species levels: score $>0 ; 4$ species level: score $\sim 0$ ) (Fig. 3). The index was lowest at intermediate richness (significant main effect; Table 2) and strongly decreased after the second disturbance in the 2 species level of the $\mathrm{pH}-\mathrm{pH}$ regime (significant richness $\times$ stressor interaction; Table 2). In Combination $\mathrm{B}$, the co-tolerance index increased with increasing species richness (significant main effect; Table 2) because the average score at the 2 species level (less than -0.5) pointed towards stressor-induced community sensitivity, whereas average scores at the 4 and 8 species levels were just below zero, indicating randomly distributed co-tolerances.

\section{Species-specific responses in the diversity experiment}

In Combination A, Sce dominated $B_{\text {tot }}$ of all experimental communities $(2,4$, and 8 species levels) throughout the entire experiment (see Supplements $6 \& 7$ at www.int-res.com/articles/suppl/b017p197_ supp.pdf for details). Higher dominance of Sce was correlated to higher $B_{\text {tot }}$ i.e. higher evenness in the presence of a high-performing species resulted in lower biomass production (Fig. 4A).

The dominance structure was much more variable in Combination B, differing among species levels and with stressor regime (see Supplements $6 \& 7$ for

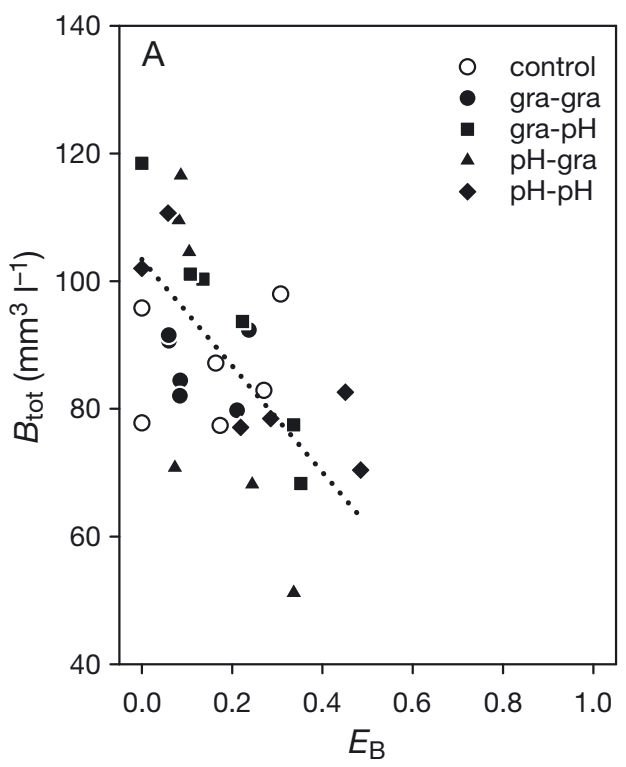

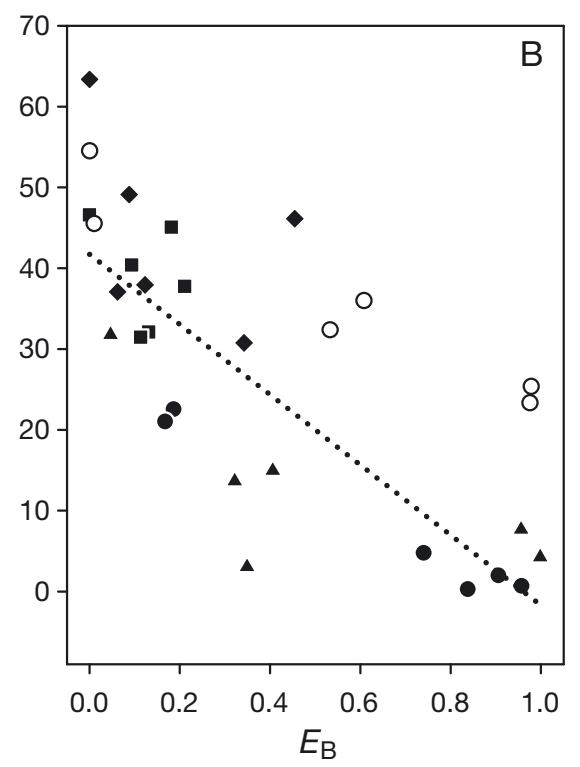

Fig. 4. Relationship between evenness $\left(E_{\mathrm{B}}\right)$ and total biovolume $\left(B_{\text {tot }}\right)$ at the end of the diversity experiment. Data for disturbed and undisturbed control treatments are shown. Correlation analyses are based on communities affected by multiple stressors (grazing [gra] and $\mathrm{pH})$. (A) Species combination A (correlation: $r=0.668$ ); (B) species combination $\mathrm{B}$ (correlation: $r=0.804)$. The dotted regression line shows the slope of the relationship 
details). At the end of the experiment, Mel and Cry co-dominated the 2 species level of the controls, Mel dominated the 4 species level with Cry being abundant and $C y l$ was highly dominant at the 8 species level. Prior to stressor application, $\mathrm{Mel}$ dominated at the 2 and 4 species levels, and Fra contributed most of $B_{\text {tot }}$ at the 8 species level. After the application of the stressor regimes, a complex pattern between richness and the stressor regimes appeared. In the 2 and 4 species levels, Mel remained dominant after $\mathrm{pH}-\mathrm{pH}$ and gra-pH disturbance sequences. Under pH-gra and gra-gra regimes, Cry became dominant in the 2 species level, and $C y c$ became dominant under the gra-gra regime in the 4 species level. The 8 species level shifted from Fra dominance to $C y l$ under the gra-gra and $\mathrm{pH}$-gra regimes, whereas Fra retained dominance under the $\mathrm{pH}-\mathrm{pH}$ and gra- $\mathrm{pH}$ regimes. In Combination $B$, evenness was also negatively correlated to $B_{\text {tot }}$, but, in the absence of a high-performing species, biomass production was low (Fig. 4B).

\section{DISCUSSION}

\section{Species performance in single and mixed cultures}

The monoculture experiment revealed speciesspecific differences concerning the traits initial growth rate, competitive ability, as well as biovolume loss and recovery rate in response to the stressors $\mathrm{pH}$ reduction and grazing. Although some species were functionally similar with regard to biovolume production (indicating redundancy sensu Walker 1992), these species differed in resource use, biovolume loss and recovery rate. Thus, trait variance in the diversity experiment was certain to increase with increasing species richness.

Sce was identified as the best-performing species. It had the highest initial growth rate, a high competitive ability for limiting nutrient phosphorus, low biovolume losses in response to the stressors $\mathrm{pH}$ reduction and grazing, and it recovered quickly from the disturbance caused by those stressors. This species was included in the inoculum of each richness level of Combination A and was absent in Combination B. In the diversity experiment, Sce became dominant regardless of the richness level of the experimental communities. Consequently, $E_{\mathrm{B}}$ in Combination $\mathrm{A}$ was generally low, but it increased with increasing species richness. This was mainly due to $C h l$, another productive species that became subdominant at higher richness levels in Combination A.
While Sce performed as expected in mixed culture, the performance of other species in mixed culture differed from their performance in the monoculture experiments. $\mathrm{Chl}$ for example became subdominant at the 2 and 4 species levels, although Ped had displayed lower losses and higher recovery rates in response to single stressors in the monoculture experiment. Such discrepancies in species responses to single and multiple stressors are likely to be due to interactions that cause non-additive effects (Breitburg et al. 1998, Vinebrooke et al. 2004). In the communities of Combination $\mathrm{B}, \mathrm{Mel}$ performed better at the 2 species level than at the 4 and 8 species levels, indicating that a species' ability to tolerate multiple stressors can depend on the competitive surrounding (Vinebrooke et al. 2004).

Moreover, not only the type of stressor but also the temporal sequences affected the response, as mixtures in both species combinations responded differently to $\mathrm{pH}$-gra compared to gra-pH sequences. Previously, Fukami (2001) found that community composition diverges with different sequences of the same type and intensity of disturbance. Obviously, the ability to cope with a certain stressor depends strongly on the disturbance history, which lends support to the idea of community co-tolerance or sensitivity, i.e. the change in a community's susceptibility to disturbance by shifts in community composition (Vinebrooke et al. 2004).

\section{Final biovolume and temporal variation in biovolume}

Both artificial disturbances were highly effective, as we observed up to $50 \%$ loss of biovolume in the monocultures (Supplement 2) and an average of $35 \%$ loss of biovolume after $\mathrm{pH}$ reduction and $37 \%$ loss after grazing applications in the diversity experiment. Indeed, both stressors increased the temporal variation of total biovolume compared to the control. The monoculture experiment showed that species' responses to the different disturbances was uncorrelated in our species pool.

In Combination A, community performance depended strongly on Sce alone, and any decrease in the relative proportion of this species reduced biomass production and stability. Thus, adding more species reduced stability, and higher evenness was related to lower $B_{\text {tot }}$. In fact, the presence of Sce inhibited the presence of long-lasting stressor effects on $B_{\text {tot }}$. Thus, Combination A corresponds well to the model settings of Norberg et al.'s (2001) study of the relationship between trait variance and productivity 
in stable and variable environments. Contradicting prevalent ecosystem theory, according to which niche differentiation increases production in stable environments (Tilman et al. 1997, Hector et al. 1999), Norberg et al. (2001) argued that a single species with certain optimal traits allows the highest productivity under stable environmental conditions. An increase in trait variance reduces productivity, because species are present that perform suboptimally under the given environmental conditions. The model predicts that long-term productivity in variable environments increases with trait variance, because species are present that are able to respond to environmental changes. However, in our experiment, the most productive species was also the one species promoting stability, thereby preventing that an increase in trait variance with higher species richness increased biovolume production in the disturbed treatments.

Communities without Sce produced less biomass, and higher $B_{\text {tot }}$ was observed in Combination A than in B across all richness levels. Given our experimental design, we cannot generalise beyond the compositions tested here. However, the importance of species identity effects in biodiversity-production relationships is frequently found and often related to trait combinations (for a discussion see Hillebrand \& Matthiessen 2009). The presence of such 'super-species' that show high performance for multiple situations is a frequent phenomenon in experiments with a small species pool and low complexity of physical or biological structure (Weis et al. 2008, Ptacnik et al. 2010).

When communities without this high-performing species (Combination B) were exposed to multiple stressors, there was no consistent relationship of $B_{\text {tot }}$ and $E_{\mathrm{B}}$ to the different levels of species richness. $B_{\mathrm{tot}}$ increased and $E_{\mathrm{B}}$ decreased with species richness under those stressor regimes that were connected with an overall low final biovolume and high biovolume variability (gra-gra and $\mathrm{pH}-\mathrm{gra}$ ). $\mathrm{Cyl}$ dominated at the highest level of species richness in these treatments. When multiple stressors decreased $B_{\text {tot }}$ only marginally compared to the control, $B_{\text {tot }}$ decreased $(\mathrm{pH}-\mathrm{pH})$ and $E_{\mathrm{B}}$ increased $(\mathrm{pH}-\mathrm{pH}$, gra- $\mathrm{pH})$ with species richness. Here Fra dominated at the highest richness level. Increasing richness (i.e. trait variance) increased the biovolume production of communities only in certain stressor combinations, a situation which was caused by local dominance rather than by niche separation or facilitation, since high levels of $B_{\text {tot }}$ in species-rich treatments were connected with low levels of $E_{\mathrm{B}}$ and vice versa (Doak et al. 1998, Cottingham et al. 2001). This emphasises the importance of addressing not only richness, but also spe- cies identity and dominance when investigating biodiversity effects in general (Wilsey et al. 2005, Hillebrand et al. 2008), and DSH especially. Moreover, our results indicate that the effect of response diversity is context dependent, as the positive effect of richness on $B_{\text {tot }}$ depends on the stressor regime.

Richness did not reduce $\mathrm{CV}_{\mathrm{B}}$ in either Combination $\mathrm{A}$ or $\mathrm{B}$. By contrast, $\mathrm{CV}_{\mathrm{B}}$ increased with richness in $\mathrm{A}$ and in some stressor sequences of $B$. The effect of richness on stability was thus not expressed as a buffering of the temporal variability but rather by providing fast recovery (see below). However, the presence of $S c e$ reduced $\mathrm{CV}_{\mathrm{B}}$ (cf. Combinations A and B in Fig. 1); thus, the presence of a high-performing species maintained low variability. Similarly, Steiner et al. (2005) found the dominance of fastgrowing green algae (Ankistrodesmus sp. and Chlorella sp.) to be negatively related to community biovolume variability.

\section{Resistance and resilience to multiple stressors}

In Combination B biovolume loss tended to be higher and the recovery rate lower than in A. Resistance increased with increasing species richness when a high-performing species was present at all richness levels (Combination A), whereas the opposite was true in the absence of a high-performing species (Combination B). When Sce was present, it remained dominant under the impact of disturbance and carried the post-disturbance production. At higher richness levels, sub-dominant and abundant species also continued to grow, explaining the positive richness effect in Combination A. Similar to natural systems (O'Neill 1999), resistance to multiple stressors in our experiment was a function of immediate losses to both stressors, and, since the stressors were applied with a $7 \mathrm{~d}$ time lag, recovery from the stressor that was applied first. Highest losses were connected to communities where dominant, fastgrowing species after the first stress event either stopped growing or continued to grow at only a very low rate. Compensatory growth could be observed in species that had performed poorly before the first stressor was applied, and thus had started out from a comparatively low specific biovolume.

Regardless of the differences in dominance structure development, we found evidence for stressorinduced community tolerance in both species combinations. The co-tolerance index was high in the presence of Sce, whereas, though low, it increased with species richness in the absence of Sce. Thus, species 
traits determining the tolerance to one stressor can influence how communities respond to other stressors (Vinebrooke et al. 2004). Stressor-induced species tolerances result in antagonistic community responses to multiple stressors and can explain the tendency towards the antagonistic multiple stressor effects often found in autotrophic communities (Vinebrooke et al. 2004, Crain et al. 2008).

Resilience was more strongly affected by the stressor regime than by species richness, which showed no significant main effect. The recovery rates tended to be higher in treatments that had experienced high losses in both species combinations. A potential explanation for this pattern is a 'self-regulation' of the steady state, which is one of the characteristics of the semi-continuous culture technique that we used in our experiments (Monod 1950). In the absence of disturbance, loss and growth rates equal zero at steady state. If disturbance reduces total biovolume, the resulting reduction in resource use causes resource concentrations to increase, which initiates the recovery of the community. The more biovolume is removed, the more resources become available and the higher the recovery rate (Flöder \& Sommer 2006).

The effect of richness on resilience was embedded in significant richness $\times$ stressor interactions in both species combinations. With Sce, recovery rates increased with species richness under those stressor regimes that led to small or moderate losses, whereas they decreased with richness when biovolume loss was high. The latter was presumably connected to higher resource availability, allowing $S c e$ to respond with high growth rates leading to faster recoveryan effect diluted by more species being present. When biomass losses were low, recovery was driven by more species showing compensatory growth, leading to increased resilience with increased richness. This interaction corresponds to the results of a study on diversity effects on stability of multitrophic communities (Steiner et al. 2005, 2006), where resilience was positively related to diversity at low productivity, but not at high productivity. Increasing resilience was related to the increasing dominance of fast-growing species of small green algae. In randomly assembled communities, resilience was related to the functional trait of the species present. When the same species that had dominated before the stressors were applied dominated at the end of the experiment, recovery rate decreased with richness. When the dominance structure changed continuously and the dominant species did not recover from the multiple stressors, recovery rates were either low at all richness levels or increased with species richness.

\section{Conclusions}

Both stressors, $\mathrm{pH}$ reduction and grazing, play important roles in natural systems. Episodic acid stress threatens freshwater ecosystems in acidified regions of Europe and northern America, because phases of droughts and floods are expected to intensify due to climatic change. Drawdown of the water table in water-logged organic soils cause previously reduced compounds to become re-oxidised, which results in pulses of acidic, sulphaterich water (Devito et al. 1999, Laudon et al. 2004). The artificial grazing that we employed in our experiments consisted of a rather unspecific removal of microalgae. In natural systems grazing can affect phytoplankton communities in different ways. Grazers can be general in their feeding behaviour, or they can be specialised feeders (Hillebrand \& Shurin 2005). The rapidly increasing grazing pressure of a growing Daphnia spp. population on small-celled phytoplankton species in spring, resulting in the clear water stage of northern temperate lakes, is a well researched phenomenon (e.g. Sommer et al. 1986). Since Sce is prone to grazing by Daphnia spp., this type of grazing would have affected the ranking of species performance and community development in our study. This fact emphasises that the concept of super-species depends on species traits and stressor identity. 'Super-species' occur in several phytoplankton biodiversityecosystem functioning experiments, especially under highly controlled culture conditions (Ptacnik et al. 2010). Natural fluctuations in real ecosystems will potentially reduce the role of such species, as the increased potential niche space allows for a variety of successful trait combinations rather than only one. Isbell et al. (2011) showed that in fact more species contribute to the ecosystem functioning of environments that change in space or time than those in constant environments.

The interdependence of stressor regimes, species traits and species richness determines which mechanisms stabilise natural communities. If dominant species remain the best performers regardless of disturbance, stability will depend on the population dynamics of species with these successful functional traits. If, on the other hand, disturbance or environmental change reverses the hierarchy of successful functional traits and dominant species become rare or lost (Jablonski 1994, Grime 1998), compensatory growth of rare species occurs. In natural systems, such an effect could insure communities against complete failure. 
Acknowledgements. We thank M. Eaton for proof reading; H. Tiebel and A. Pasternak for laboratory assistance; W. Lampert for the Scenedesmus obliquus strain; and M. and B. Melkonian for all other cultures. Funding was by the Deutsche Forschungsgemeinschaft (DFG Hi 848/1-2).

\section{LITERATURE CITED}

Breitburg DL, Baxter JW, Hatfield CA, Howarth RW and others (1998) Understanding effects of multiple stressors: ideas and challenges. In: Pace ML, Groffman PM (eds) Successes, limitations, and frontiers in ecosystem science. Springer, Berlin, p 416-431

Cardinale BJ, Srivastava DS, Dufly JE, Wright JP, Downing AL, Sankaran M, Jouseau C (2006) Effects of biodiversity on the functioning of trophic groups and ecosystems. Nature 443:989-992

Cottingham KL, Brown BL, Lennon JT (2001) Biodiversity may regulate the temporal variability of ecological systems. Ecol Lett 4:72-85

Crain CM, Kroeker K, Halpern BS (2008) Interactive and culmulative effects of multiple human stressors in marine systems. Ecol Lett 11:1304-1315

Devito KJ, Hill AR, Dillon PJ (1999) Episodic sulphate export form wetlands in acidified headwater catchments: prediction at the landscape scale. Biogeochemistry 44: 187-203

Doak DF, Bigger D, Harding EK, Marvier MA, O'Malley RE, Thomson D (1998) The statistical inevitability of stability-diversity relationships in community ecology. Am Nat 151:264-276

> Downing AL (2008) Environmental fluctuations induce scaledependent compensation and increase stability in plankton ecosystems. Ecology 89:3204-3214

Fine PVA, Miller ZJ, Mesones I, Irazuzta S and others (2006) The growth-defense trade-off and habitat specialization by plants in Amazonian forests. Ecology 87:150-162

Flöder S, Burns CW (2005) The influence of fluctuating light on diversity and species number of nutrient limited phytoplankton. J Phycol 41:950-956

Flöder S, Sommer U (2006) Competition of aquatic microalgae in variable environments: the disturbance effect. In: Subba Rao SV (ed) Algal cultures, analogues of blooms and applications. Science Publishers, Enfield, p 533-569

> Fukami T (2001) Sequence effects of disturbance on community structure. Oikos 92:215-224

> Gonzalez A, Descamps-Julien B (2004) Population and community variability in randomly fluctuating environments. Oikos 106:105-116

Grime JP (1979) Plant strategies and vegetation processes. John Wiley \& Sons, Chichester

$>$ Grime JP (1998) Benefits of plant diversity to ecosystems: immediate, filter and founder effects. J Ecol 86:902-910

Guillard RRL (1975) Culture of phytoplankton for feeding marine invertebrates. In: Smith WL, Chantey MH (ed) Culture of marine invertebrate animals. Plenum Publishers, New York, NY, p 29-60

Hector A, Schmid B, Beierkuhnlein C, Caldeira MC and others (1999) Plant diversity and productivity experiments in European grasslands. Science 286:1123-1127

Hillebrand H, Matthiessen B (2009) Biodiversity in a complex world: consolidation and progress in functional biodiversity research. Ecol Lett 12:1405-1419

Hillebrand H, Shurin JB (2005) Biodiversity and aquatic food webs. In: Belgrano A, Scharler UM, Dunne JB, Ulanowicz RE (eds) Aquatic food webs. Oxford University Press, New York, NY, p 184-197

Hillebrand H, Dürselen CD, Kirschtel D, Pollingher U, Zohary T (1999) Biovolume calculation for pelagic and benthic microalgae. J Phycol 35:403-424

> Hillebrand H, Bennett DM, Cadotte MW (2008) Consequences of dominance: a review of evenness effects on local and regional ecosystem processes. Ecology 89: 1510-1520

Houlahan JE, Currie DJ, Cottenie K, Cumming GS and others (2007) Compensatory dynamics are rare in natural ecological communities. Proc Natl Acad Sci USA 104: 3273-3277

Huston MA (1997) Hidden treatments in ecological experiments: re-evaluating the ecosystem function of biodiversity. Oecologia 110:449-460

Isbell F, Calcagno V, Hector A, Connolly J and others (2011) High plant diversity is needed to maintain ecosystem services. Nature 477:199-202

Jablonski D (1994) Extinctions in the fossil record. Philos Trans R Soc Lond B 344:11-17

> Laudon H, Dillon PJ, Eimers MC, Semkin RG, Jeffries DS (2004) Climate-induced episodic acidification of streams in central Ontario. Environ Sci Technol 38:6009-6015

Liiri M, Setälä H, Pennanen HJT, Fritze H (2002) Relationship between soil microarthropod species diversity and plant growth does not change when the system is disturbed. Oikos 96:137-149

Monod J (1950) La technique de culture continue: theorie et applications. Ann Inst Pasteur (Paris) 79:390-410

> Moorthi SI, Hillebrand H, Wahl M, Berninger UG (2008) Consumer diversity enhances secondary production by complementarity effects in experimental ciliate assemblages. Estuar Coast 31:152-162

Norberg J, Swaney DP, Dushoff J, Lin J, Casagrandi R, Levin SA (2001) Phenotypic diversity and ecosystem functioning: a theoretical framework. Proc Natl Acad Sci USA 98:11376-11381

O'Neill RV (1999) Recovery in complex ecosystems. J Aquat Ecosyst Stress Recovery 6:181-187

Pfisterer AB, Schmid B (2002) Diversity-dependent production can decrease the stability of ecosystem functioning. Nature 416:84-86

Ptacnik R, Andersen T, Brettum P, Lepistö L, Willén E (2010) Regional species pools control community saturation in lake phytoplankton. Proc Biol Sci 277:3755-3764

> Roscher C, Weigelt A, Proulx R, Marquard E, Schumacher J, Weisser WW, Schmid B (2011) Identifying populationand community-level mechanisms of diversity-stability relationships in experimental grasslands. J Ecol 99: 1460-1469

Sasaki T, Lauenroth WK (2011) Dominant species, rather than diversity, regulates temporal stability of plant communities. Oecologia 166:761-768

Sommer U, Gliwicz ZM, Lampert W, Duncan A (1986) The PEG-model of seasonal succession of planktonic events in fresh waters. Arch Hydrobiol 106:433-471

Steiner CF, Long ZT, Krumins JA, Morin PJ (2005) Temporal stability of aquatic food webs: partioning the effects of species diversity, species composition and enrichment. Ecol Lett 8:819-828

Steiner CF, Long ZT, Krumins JA, Morin PJ (2006) Population and community resilience in multitrophic communities. Ecology 87:996-1007 
Strickland JDH, Parsons TR (1978) A practical handbook of seawater analysis. Bulletin of the Fisheries Research Board of Canada, Ottawa

Tilman D (1982) Resource competition and community structure. Princeton University Press, Princeton, NJ

Tilman D (1996) Biodiversity: population versus ecosystem stability. Ecology 77:350-363

Tilman D, Lehman CL, Thomson KT (1997) Plant diversity and ecosystem productivity: theoretical considerations. Proc Natl Acad Sci USA 94:1857-1861

Valone TJ, Barber NA (2008) An empirical evaluation of the insurance hypothesis in diversity-stability models. Ecology 89:522-531

van Ruijven J, Berendse F (2010) Diversity enhances community recovery, but not resistance, after drought. J Ecol 98:81-86

Vasseur DA, Gaedke U (2007) Spectral analysis unmasks synchronous and compensatory dynamics in plankton communities. Ecology 88:2058-2071

Vinebrooke RD, Cottingham KL, Norberg J, Scheffer M, Dodson SI, Maberly SC, Sommer U (2004) Impacts of

Editorial responsibility: Max Tilzer,

Konstanz, Germany multiple stressors on biodiversity and ecosystem functioning: the role of species co-tolerance. Oikos 104: 451-457

Walker BH (1992) Biodiversity and ecological redundancy. Conserv Biol 6:18-23

Washington HG (1984) Diversity, biotic and similarity indices. Water Res 18:653-694

Weis JJ, Madrigal DS, Cardinale BJ (2008) Effects of algal diversity on the production of biomass in homogeneous and heterogeneous nutrient environments: a microcosm experiment. PLoS ONE 3:e2825

Wilsey BJ, Chalcraft DR, Bowles CM, Willig MR (2005) Relationships among indices suggest that richness is an incomplete surrogate for grassland biodiversity. Ecology 86:1178-1184

Winfree R, Kremen C (2009) Are ecosystem services stabilized by differences among species? A test using crop pollination. Proc Biol Sci 276:229-237

Yachi S, Loreau M (1999) Biodiversity and ecosystem productivity in a fluctuating environment: the insurance hypothesis. Proc Natl Acad Sci USA 96:1463-1468

Submitted: December 29, 2011; Accepted: October 10, 2012 Proofs received from author(s): December 4, 2012 